\title{
PERIODS OF PERIODIC POINTS OF MAPS OF THE CIRCLE WHICH HAVE A FIXED POINT
}

\author{
LOUIS BLOCK
}

\begin{abstract}
For a continuous map $f$ of the circle to itself, let $P(f)$ denote the set of positive integers $n$ such that $f$ has a periodic point of (least) period $n$. Results are obtained which specify those sets, which occur as $P(f)$, for some continuous map $f$ of the circle to itself having a fixed point. These results extend a theorem of Sarkovskii on maps of the interval to maps of the circle which have a fixed point.
\end{abstract}

1. Introduction. This paper extends the theorem of Šarkovskii on maps of the interval to maps of the circle which have a fixed point.

Let $R$ denote the real line, $I$ a closed bounded interval on $R$, and $S^{1}$ the circle. Let $C^{0}(X, Y)$ denote the set of continuous maps from $X$ to $Y$. For $f \in C^{0}(I, R)$ or $f \in C^{0}\left(S^{1}, S^{1}\right)$ let $P(f)$ denote the set of positive integers $n$ such that $f$ has a periodic point of (least) period $n$.

Let $N$ denote the set of positive integers and let $\Delta$ denote the ordering of $N$ :

$3 \Delta 5 \Delta 7 \Delta \cdots \Delta 2 \cdot 3 \Delta 2 \cdot 5 \Delta \cdots \Delta 2^{2} \cdot 3 \Delta 2^{2} \cdot 5 \Delta \cdots \Delta 2^{3} \Delta 2^{2} \Delta 2 \Delta 1$. The following theorem is proved in [2], [3] and [4].

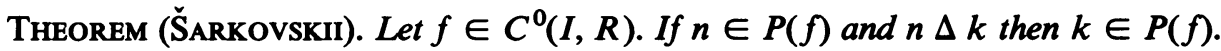
Conversely, suppose $S \subset N$ with the property that if $n \in S$ and $n \Delta k$ then $k \in S$. Then there is a map $f \in C^{0}(I, I)$ with $P(f)=S$.

Note that the theorem of Šarkovskii completely specifies those subsets of $N$ which occur as $P(f)$ for some $f \in C^{0}(I, R)$. In this paper we do the same for $f \in C^{0}\left(S^{1}, S^{1}\right)$ having a fixed point. Let $\Delta$ denote the ordering defined above, and let $<$ denote the usual ordering of $N$. The main result of this paper is the following.

Theorem A. Let $f \in C^{0}\left(S^{1}, S^{1}\right)$. Suppose $1 \in P(f)$ and $n \in P(f)$ for some integer $n>1$. Then (at least) one of the following holds.

(i) For every integer $m$ with $n<m, m \in P(f)$.

(ii) For every integer $m$ with $n \Delta m, m \in P(f)$.

We remark that in [2] the periodic points and topological entropy of maps $f \in C^{0}\left(S^{1}, S^{1}\right)$ are studied by examining separately the four cases where the degree of $f$ is $0,1,-1$, or of absolute value greater than 1 . The results of [2] imply that Theorem A holds in all cases except where the degree of $f$ is -1 and $n$ is even. The

Received by the editors May 21, 1980 and, in revised form, October 21, 1980; presented to the Society, January 7, 1981.

1980 Mathematics Subject Classification. Primary 54H20. 
proof of Theorem A given here treats maps of all degrees simultaneously (including the case left open in [2]), using ideas from [1] and [2].

Let $f \in C^{0}\left(S^{1}, S^{1}\right)$ and suppose $f$ has degree -1 . One of the results of [2] states that if $n \in P(f)$ and $n$ is odd then statement (ii) (in Theorem A) must hold. Now, suppose $n \in P(f)$ and $n$ is even. By Theorem A, either (i) or (ii) holds. Suppose (i) holds. Then $(n+1) \in P(f)$. Since $n+1$ is odd, the result of [2] implies that $m \in P(f)$ for every positive integer $m$ with $(n+1) \Delta m$. Since $(n+1) \Delta n$, (ii) holds. Hence, we have the following.

Corollary B. Let $f \in C^{0}\left(S^{1}, S^{1}\right)$ and suppose $f$ has degree -1. If $n \in P(f)$ then $m \in P(f)$ for every integer $m$ with $n \Delta m$.

The final result of this paper is the following.

Theorem C. Let $S \subset N$ with $1 \in S$. Suppose that for every $n \in S$ with $n>1$ (at least) one of the following holds.

(i) For every integer $m$ with $n<m, m \in S$.

(ii) For every integer $m$ with $n \Delta m, m \in S$. Then there is a map $f \in C^{0}\left(S^{1}, S^{1}\right)$ such that $P(f)=S$.

The proof of Theorem $\mathrm{C}$ is obtained by using an example from [1] for $f \in$ $C^{0}\left(S^{1}, S^{1}\right)$ with $P(f)=\{1\} \cup\{k \in N: k>n\}$. This example is modified to include an invariant interval on $S^{1}$ with periodic points as specified by the theorem of Šarkovskii. Note that the example constructed has degree one. It follows from Corollary B and the results of [2] that this is the only possible degree.

2. Preliminary definitions and results. Let $f \in C^{0}\left(S^{1}, S^{1}\right)$. Let $f^{0}$ denote the identity map of $S^{1}$, and for any $n \in N$ define $f^{n}$ inductively by $f^{n}=f \circ f^{n-1}$.

Let $x \in S^{1}$. We say $x$ is a fixed point of $f$ if $f(x)=x$. If $x$ is a fixed point of $f^{n}$, for some $n \in N$, we say $x$ is a periodic point of $f$. In this case the smallest element of $\left\{n \in N: f^{n}(x)=x\right\}$ is called the period of $x$.

We define the orbit of $x$ to be $\left\{f^{n}(x): n=0,1,2, \ldots\right\}$. If $x$ is a periodic point of $f$ of period $n$, we say the orbit of $x$ is a periodic orbit of period $n$. In this case the orbit of $x$ contains exactly $n$ points, each of which is a periodic point of period $n$.

We will use the following notation throughout this paper.

Notation. Let $a \in S^{1}$ and $b \in S^{1}$ with $a \neq b$. We write $[a, b],(a, b),(a, b]$, or $[a, b)$ to denote the closed, open, or half-open interval from a counterclockwise to $b$.

We will also use the following definition.

Definition. Let $I$ and $J$ be proper closed intervals on $S^{1}$ and let $f \in C^{0}\left(S^{1}, S^{1}\right)$. We say I f-covers $J$ if, for some closed interval $K \subset I, f(K)=J$.

We conclude this section by stating three lemmas from [1] which will be used in the next section.

LEMMA 1 (LEMMA 1 OF [1]). Let $I=[a, b]$ be a proper closed interval on $S^{1}$ and let $f \in C^{0}\left(S^{1}, S^{1}\right)$. Suppose $f(a)=c$ and $f(b)=d$ and $c \neq d$. Then either $I f$-covers $[c, d]$ or I f-covers $[d, c]$.

LEMMA 2 (LEMMA 2 OF [1]). Let I and J be proper closed intervals on $S^{1}$ such that I $f$-covers $J$. Suppose $L$ is a closed interval with $L \subset J$. Then $I f$-covers $L$. 
LemMa 3 (LemMa 7 of [1]). Let $f \in C^{0}\left(S^{1}, S^{1}\right)$ and let $P$ be a periodic orbit of period $m$ where $m>3$. Suppose that $\left\{M_{1}, \ldots, M_{k}\right\}$ is a collection of closed intervals with $2<k<m$ such that

(1) for each $j \in\{1, \ldots, k\}$, there are no elements of $P$ in the interior of $M_{j}$.

(2) If $i \neq j, M_{i}$ and $M_{j}$ have disjoint interiors.

(3) If $j \in\{2, \ldots, k\}$ the endpoints of $M_{j}$ are in $P$.

(4) If $b$ is an endpoint of $M_{1}$ then either $b \in P$ or $b$ is a fixed point of $f$.

(5) For each $j \in\{1, \ldots, k-1\}, M_{j} f$-covers $M_{j+1}$.

(6) $M_{1} f$-covers $M_{1}$ and $M_{k} f$-covers $M_{1}$. Then for any positive integer $n>k$, $n \in P(f)$.

\section{Proof of Theorem A.}

Convention. In Theorems $\mathrm{A}_{1}$ and $\mathrm{A}_{2}$ in this section, we assume that $f \in$ $C^{0}\left(S^{1}, S^{1}\right)$ and $f$ has a fixed point $e$. Also, we suppose that $f$ has a periodic orbit $P=\left\{p_{1}, \ldots, p_{n}\right\}$ of period $n \geqslant 3$ where $P \cap\left(p_{k}, p_{k+1}\right)=\varnothing$ for $k=1, \ldots, n-1$ and $P \cap\left(p_{n}, p_{1}\right)=\varnothing$. Finally, we let $I_{1}=\left[p_{1}, p_{2}\right], I_{2}=\left[p_{2}, p_{3}\right], \ldots, I_{n-1}=$ $\left[p_{n-1}, p_{n}\right]$, and $I_{n}=\left[p_{n}, p_{1}\right]$, and set $A=\left\{I_{1}, \ldots, I_{n}\right\}$.

TheORem $A_{1}$. Suppose that for each $I_{j} \in A$ there is some $I_{k} \in A$ with $k \neq j$ such that $I_{k} f$-covers $I_{j}$. Then for every integer $m$ with $n<m, m \in P(f)$.

Proof. Since the fixed point $e$ of $f$ must be in one of the intervals $I_{j} \in A$, we may assume without loss of generality that $e \in I_{n}$. We have two cases.

Case 1. $I_{n} f$-covers $I_{n}$.

Let $K_{1}=I_{n}$ and let $A_{1}=\left\{I_{j} \in A: K_{1} f\right.$-covers $\left.I_{j}\right\}$. It follows from Lemmas 1 and 2 and the fact that $P$ is a periodic orbit that $A_{1}$ contains at least one element $I_{j}$ of $A$ with $j \neq n$. Also, since $I_{n} f$-covers $I_{n}, I_{n} \in A_{1}$.

Suppose that $A_{1} \neq A$. Let $K_{2}$ denote the union of the intervals in $A_{1}$. It follows from Lemmas 1 and 2 that $K_{2}$ is connected. Hence $K_{2}$ is a proper closed interval on $S^{1}$. Let $A_{2}=\left\{I_{j} \in A: K_{2} f\right.$-covers $\left.I_{j}\right\}$. Then $A_{1} \subset A_{2}$.

We will show that $A_{2} \neq A_{1}$. First suppose there are at least two distinct elements of $A$ not in $A_{1}$. Then there is an element of $P$ not in $K_{2}$. Since $P$ is a periodic orbit, it follows (using Lemmas 1 and 2) that $A_{2} \neq A_{1}$. Now suppose there is exactly one element $I_{s}$ of $A$ not in $A_{1}$. By hypothesis, for some $I_{t} \in A$ with $t \neq s, I_{t} f$-covers $I_{s}$. Since $I_{t} \neq I_{s}, I_{t} \in A_{1}$. Hence $I_{s} \in A_{2}$, so $A_{2} \neq A_{1}$.

Now, let $A_{i}=\left\{I_{j} \in A: K_{i} f\right.$-covers $\left.I_{j}\right\}$ and let $K_{i+1}$ denote the union of the intervals in $A_{i}$. Then as above it follows that if $A_{i} \neq A$ then $A_{i}$ is a proper subset of $A_{i+1}$. Thus, for some positive integer $r$ with $r<n, A_{r}=A$.

We claim that for any positive integer $i$ with $2<i<r$, if $I_{j} \in A_{i}$ then $I_{u} f$-covers $I_{j}$ for some $I_{u} \in A_{i-1}$. To see this, suppose that $I_{j} \in A_{i}$. Then since $K_{i} f$-covers $I_{j}$, $f(D)=I_{j}$ for some closed interval $D \subset K_{i}$. There is a closed interval $E \subset D$ such that $f(E)=I_{j}$ and $f$ maps the interior of $E$ to the interior of $I_{j}$. Hence, there are no elements of $P$ in the interior of $E$. Thus, $E \subset I_{u}$ for some $I_{u} \in A_{i-1}$, and the claim is established.

Now, since $A_{r}=A$, our hypothesis implies that some element of $A_{r}$ other than $I_{n}$ $f$-covers $I_{n}$. Let $w$ denote the smallest positive integer such that some element of $A_{w}$, other than $I_{n}, f$-covers $I_{n}$. Let $L_{1}$ denote an element of $A_{w}$ such that $L_{1} \neq I_{n}$ and 
$L_{1} f$-covers $I_{n}$. If $w>1$, let $L_{2}$ denote an element of $A_{w-1}$ such that $L_{2} f$-covers $L_{1}$. Continuing we obtain distinct elements of $A, L_{1}, L_{2}, \ldots, L_{w}$ with $L_{i} \in A_{w+1-i}$ for $i=1, \ldots, w$ such that $L_{1} f$-covers $I_{n}$ and $L_{i} f$-covers $L_{i-1}$ for $i=2, \ldots, w$. Let $k=w+1$, and let $M_{1}=I_{n}, M_{2}=L_{w}, M_{3}=L_{w-1}, \ldots, M_{k}=L_{1}$. Then $k \leqslant n$ and $\left\{M_{1}, \ldots, M_{k}\right\}$ is a collection of closed intervals satisfying the hypothesis of Lemma 3. Hence, by Lemma 3, $m \in P(f)$ for every integer $m>n$.

Case 2. $I_{n}$ does not $f$-cover $I_{n}$.

By continuity, $\exists x \in\left[e, p_{1}\right]$ such that $f(x) \in\left\{p_{1}, p_{n}\right\}$. Hence, $\exists a \in\left[e, p_{1}\right]$ such that $f(a) \in\left\{p_{1}, p_{n}\right\}$ and, for all $x \in(e, a), f(x) \notin\left\{p_{1}, p_{n}\right\}$. Similarly, $\exists b \in\left[p_{n}, e\right]$ such that $f(b) \in\left\{p_{1}, p_{n}\right\}$ and, for all $x \in(b, e), f(x) \notin\left\{p_{1}, p_{n}\right\}$.

Suppose that $f(a)=p_{n}$ and $f(b)=p_{1}$. Then $f([b, a])=\left[p_{n}, p_{1}\right]$. This is a contradiction since $I_{n}=\left[p_{n}, p_{1}\right]$ does not $f$-cover itself. Hence either $f(a)=p_{1}$ or $f(b)=p_{n}$. Without loss of generality we may assume that $f(a)=p_{1}$. Then (by Lemma 2) $\left[e, p_{1}\right] f$-covers $\left[e, p_{1}\right]$.

Suppose that $f(x)=p_{n}$ for some $x \in\left[e, p_{1}\right]$. By choice of $a, x \in\left(a, p_{1}\right]$. By Lemma 1 , the interval $[a, x] f$-covers either $I_{n}=\left[p_{n}, p_{1}\right]$ or $\left[p_{1}, p_{n}\right]$. Since $I_{n}$ does not $f$-cover itself, $[a, x] f$-covers $\left[p_{1}, p_{n}\right]$. Thus, $\left[e, p_{1}\right] f$-covers $\left[p_{1}, p_{n}\right]$. By Lemma 2 , $\left[e, p_{1}\right] f$-covers $I_{j}$ for every $I_{j} \in A$ with $j \neq n$. By hypothesis $I_{s} f$-covers $\left[e, p_{1}\right]$ for some $I_{s} \in A$ with $s \neq n$. Hence, the conclusion of this theorem follows from Lemma 3 (with $k=2, M_{1}=\left[e, p_{1}\right]$, and $M_{2}=I_{s}$ ). Thus, we may assume that $f(x) \neq p_{n}$ for all $x \in\left[e, p_{1}\right]$.

Now, we modify the argument of Case 1 , replacing $A=\left\{I_{1}, \ldots, I_{n}\right\}$ by $A_{0}=$ $\left\{\left[e, p_{1}\right], I_{1}, \ldots, I_{n-1}\right\}$, and starting with $K_{1}=\left[e, p_{1}\right]$ instead of $K_{1}=I_{n}$. We let $A_{i}=\left\{I \in A_{0}: K_{i} f\right.$-covers $\left.I\right\}$ and let $K_{i+1}$ denote the union of the intervals in $A_{i}$. It follows from the previous paragraph that $A_{1}=\left\{\left[e, p_{1}\right], I_{1}, \ldots, I_{t}\right\}$ for some positive integer $t$.

By hypothesis, some element of $\left\{I_{1}, \ldots, I_{n-1}\right\} f$-covers $I_{n}$. Also, since $P$ is a periodic orbit, if $A_{i}$ does not contain an interval which $f$-covers $I_{n}$ then $A_{i}$ is a proper subset of $A_{i+1}$. Hence, for some positive integer $r$ with $1<r<n-1, A_{r}$ contains an interval $I_{s} \in\left\{I_{1}, \ldots, I_{n-1}\right\}$ such that $I_{s} f$-covers $I_{n}$. By Lemma 2, $I_{s}$ $f$-covers $\left[e, p_{1}\right]$. As in Case 1 , we obtain a collection of closed intervals $\left\{M_{1}, \ldots, M_{k}\right\}$ (here $M_{1}=\left[e, p_{1}\right]$ ) with $2<k<n$, satisfying the hypothesis of Lemma 3. Hence, the conclusion of this theorem follows from Lemma 3. Q.E.D.

Theorem $\mathrm{A}_{2}$. Suppose that for some $I_{j} \in A$ there does not exist $I_{k} \in A$ with $k \neq j$ such that $I_{k} f$-covers $I_{j}$. Then for every positive integer $m$ with $n \Delta m, m \in P(f)$.

Proof. Let $I_{j}$ be as in the hypothesis and let $K$ denote the closure of the complement of $I_{j}$ in $S^{1}$. Let $h: K \rightarrow I$ be a homeomorphism from $K$ onto a closed interval $I$ on the real line.

Our hypothesis implies that there is a continuous map $g: I \rightarrow R$ such that, for all $x \in K, f(x) \in K$ if and only if $g(h(x)) \in I$ and in this case $h(f(x))=g(h(x))$. Thus, since the restriction of $f$ to $K$ has a periodic orbit of period $n, n \in P(g)$. By the theorem of Šarkovskii, $m \in P(g)$ for every positive integer $m$ with $n \Delta m$. Hence, $m \in P(f)$ for every positive integer $m$ with $n \Delta m$. Q.E.D.

Theorem $A$ follows immediately from Theorems $A_{1}$ and $A_{2}$. 


\section{Proof of Theorem C.}

LEMMA 4. Let $I=[a, b]$ be an interval on the real line, and let $k$ be $a$ positive integer. Let $S=\{k\} \cup\{j \in N: k \Delta j\}$. There is a map $g \in C^{0}(I, I)$ such that $g(a)=a, g(b)=b$, and $P(g)=S$.

Proof. Let $c$ and $d$ be points in $I$ with $a<c<d<b$. By the theorem of Sarkovskii, there is a continuous map $g_{0}:[c, d] \rightarrow[c, d]$ such that $P\left(g_{0}\right)=S$. There is a unique $g \in C^{0}(I, I)$ such that $g(a)=a, g(b)=b, g(x)=g_{0}(x)$ for all $x \in$ $[c, d]$, and $g$ is linear on each of the intervals $[a, c],[d, b]$. Clearly $P(g)=P\left(g_{0}\right)=$ S. Q.E.D.

Theorem C. Let $S \subset N$ with $1 \in S$. Suppose that for every $n \in S$ with $n>1$ (at least) one of the following holds:

(i) For every integer $m$ with $n<m, m \in S$.

(ii) For every integer $m$ with $n \Delta m, m \in S$.

Then there is a map $f \in C^{0}\left(S^{1}, S^{1}\right)$ such that $P(f)=S$.

Proof. Let $S \subset N$ which satisfies the hypothesis. Suppose that, for all $n \in S$, $\{k \in N: n<k\}$ is not a subset of $S$. Then for all $n \in S, k \in S$ for every integer $k$ with $n \Delta k$. By the theorem of Sarkovskii, there is a map $g \in C^{0}(I, I)$ such that $P(g)=S$. Hence, we can extend $g$ to a map $f \in C^{0}\left(S^{1}, S^{1}\right)$ with $P(f)=S$.

Thus, we may assume that, for some $n \in S,\{k \in N: n<k\} \subset S$. We may choose $n$ such that $\{k \in N: n<k\} \subset S$ but if $m<n,\{k \in N: m<k\}$ is not a subset of $S$. If $n=1$ then $S=N$ and there are maps $f \in C^{0}\left(S^{1}, S^{1}\right)$ with $P(f)=N$. Hence we may assume that $n>1$. Since $1 \in S$, this implies $n>3$.

Let $p_{1}, p_{2}, \ldots, p_{n}$ be distinct points on $S^{1}$ such that if $P=\left\{p_{1}, p_{2}, \ldots, p_{n}\right\}$ then $\left(p_{i}, p_{i+1}\right) \cap P=\varnothing$ for $i=1, \ldots, n-1$ and $\left(p_{n}, p_{1}\right) \cap P=\varnothing$. Let $e_{2} \in\left(p_{n}, p_{1}\right)$ and let $e_{1} \in\left(p_{n}, e_{2}\right)$.

We construct $f \in C^{0}\left(S^{1}, S^{1}\right)$ as follows. Let $f\left(p_{i}\right)=p_{i+1}$ for $i=1, \ldots, n-1$ and $f\left(p_{n}\right)=p_{1}$. Let $f\left(e_{1}\right)=e_{1}$ and $f\left(e_{2}\right)=e_{2}$. For $i=1, \ldots, n-2$, let $f$ map the interval $\left[p_{i}, p_{i+1}\right]$ homeomorphically onto $\left[p_{i+1}, p_{i+2}\right]$. Let $f$ map $\left[p_{n-1}, p_{n}\right]$ homeomorphically onto $\left[p_{n}, p_{1}\right]$. Also, let $f$ map $\left[p_{n}, e_{1}\right]$ homeomorphically onto $\left[e_{1}, p_{1}\right]$ and let $f$ map $\left[e_{2}, p_{1}\right]$ homeomorphically onto $\left[e_{2}, p_{2}\right]$.

It remains to define $f$ on $\left[e_{1}, e_{2}\right]$. Let $T=\{i \in S: i<n\}$. Note that $T \neq \varnothing$ since $1 \in T$. There is a unique element $k$ of $T$ such that, for all $i \in T$ with $i \neq k, k \Delta i$. By Lemma 4, there is a map $g \in C^{0}\left(\left[e_{1}, e_{2}\right],\left[e_{1}, e_{2}\right]\right)$ with $g\left(e_{1}\right)=e_{1}, g\left(e_{2}\right)=e_{2}$ and $P(g)=\{k\} \cup\{j \in N: k \Delta j\}$. Define $f$ on $\left[e_{1}, e_{2}\right]$ by $f(x)=g(x)$ for $x \in\left[e_{1}, e_{2}\right]$. Thus we have constructed $f \in C^{0}\left(S^{1}, S^{1}\right)$.

By construction $e_{1}$ and $e_{2}$ are fixed points of $f$ and $\left\{p_{1}, p_{2}, \ldots, p_{n}\right\}$ is a periodic orbit of period $n$. It follows from Theorem $A_{1}$ that $m \in P(f)$ for every integer $m$ with $m>n$. Also, by construction, all periodic points outside the interval $\left[e_{1}, e_{2}\right]$ have period at least $n$.

Thus $P(f)=\{m \in N: n<m\} \cup\{k\} \cup\{m \in N: k \Delta m\}=S$. Q.E.D. 


\section{REFERENCES}

1. L. Block, Periodic orbits of continuous mappings of the circle, Trans. Amer. Math. Soc. 260 (1980), 553-562.

2. L. Block, J. Guckenheimer, M. Misiurewicz and L. S. Young, Periodic points and topological entropy of one dimensional maps, Global Theory of Dynamical Systems, Proceedings (Northwestern, 1979), Lecture Notes in Math., vol. 819, Springer-Verlag, Berlin and New York, 1980, pp. 18-34.

3. A. N. Sarkovskii, Coexistence of cycles of a continuous map of a line into itself, Ukrain. Mat. $\grave{Z} .16$ (1964), 61-71.

4. P. Stefan, A theorem of Sarkouskii on the existence of periodic orbits of continuous endomorphisms of the real line, Comm. Math. Phys. 54 (1977), 237-248.

Department of Mathematics, University of Florida, Gainesville, Florma 32611 